\title{
HOMOLOGICAL APPROACH TO THE HERNANDEZ-LECLERC CONSTRUCTION AND QUIVER VARIETIES
}

\author{
GIOVANNI CERULLI IRELLI, EVGENY FEIGIN, AND MARKUS REINEKE
}

\begin{abstract}
In a previous paper the authors have attached to each Dynkin quiver an associative algebra. The definition is categorical and the algebra is used to construct desingularizations of arbitrary quiver Grassmannians. In the present paper we prove that this algebra is isomorphic to an algebra constructed by Hernandez-Leclerc defined combinatorially and used to describe certain graded Nakajima quiver varieties. This approach is used to get an explicit realization of the orbit closures of representations of Dynkin quivers as affine quotients.
\end{abstract}

\section{INTRODUCTION}

Let $Q$ be a Dynkin quiver and let $k$ be an algebraically closed field of characteristic zero. In $[8]$ the authors have defined an algebra $B_{Q}$. The definition of $B_{Q}$ is of categorical nature and in principle can be applied not only to the path algebra of a Dynkin quiver, but to any algebra with finite number of indecomposable representations. The algebra $B_{Q}$ plays a crucial role in the construction of desingularizations of quiver Grassmannians for the quiver $Q$ (see also [7, [10]). Roughly speaking, given a representation $M$ of the path algebra $k Q$ and a dimension vector e, one can construct a $B_{Q}$-module $\widehat{M}$ and a dimension vector $\widehat{\mathbf{e}}$ of the Gabriel quiver of $B_{Q}$. Then the quiver Grassmannian $\operatorname{Gr}_{\mathbf{e}}(M)$ can be desingularized by means of the quiver Grassmannian $\operatorname{Gr}_{\widehat{\mathbf{e}}}(\widehat{M})$ of the algebra $B_{Q}$ (if $\operatorname{Gr}_{\mathbf{e}} M$ is not irreducible, then one needs several such quiver Grassmannians). A central role in the definition of $B_{Q}$ is played by a certain category of embeddings between projective modules of $Q$. This approach allows usage of homological tools for the study of the geometry of various objects related to a quiver, such as representation varieties, orbit closures, quiver Grassmannians, etc. In this paper we continue the study of the algebras $B_{Q}$. Let us briefly describe our main results.

Let $\widehat{Q}$ be the Gabriel quiver of the algebra $B_{Q}$. In [8] the quiver $\widehat{Q}$ was described in terms of the representation theory of $k Q$. For example, $\widehat{Q}$ has two types of vertices: vertices of the first type are labelled by the non-projective indecomposable $k Q$-modules, and vertices of the second type are in one-to-one correspondence with the vertices of the initial quiver $Q$. The arrows of $\widehat{Q}$ can be also explicitly described in representation-theoretic terms. By definition, there exists an admissible ideal $I$ such that $B_{Q}=k \widehat{Q} / I$. One of our goals is to describe the ideal $I$.

In [11, Section 9], Hernandez and Leclerc introduced an algebra $\widetilde{\Lambda}_{Q}$, defined by an explicit combinatorial description of its Gabriel quiver $\widetilde{\Gamma}_{Q}$ and by an explicit set of relations. This algebra was used to provide a realization of certain graded Nakajima

Received by the editors March 13, 2013 and, in revised form, October 17, 2013.

2010 Mathematics Subject Classification. Primary 14L30, 14M15, 16G20, 18F99. 
quiver varieties via the representation varieties of the algebra $\widetilde{\Lambda}_{Q}$. More precisely, the graded quiver varieties in question are isomorphic to the representation varieties $R_{\mathbf{d}}(Q)$ of the quiver $Q$, and they are realized as affine quotients of representation varieties of $\widetilde{\Lambda}_{Q}$. Our first result is:

Theorem 1.1. The algebras $B_{Q}$ and $\widetilde{\Lambda}_{Q}$ are isomorphic. In particular, the quiver $\widehat{Q}$ is isomorphic to $\widetilde{\Gamma}_{Q}$.

We also rederive the Hernandez-Leclerc realization of the representation varieties $R_{\mathbf{d}}(Q)$ of the quiver $Q$ as affine quotients of representation varieties of $B_{Q}$. Actually, our approach allows us to strengthen the results of [11. Namely, in [11, Section 9], the authors show that not only the representation varieties $R_{\mathbf{d}}(Q)$ are isomorphic to certain graded quiver varieties, but moreover the stratification of $R_{\mathbf{d}}(Q)$ by the orbits of the structure group $G_{\mathbf{d}}$ coincides with the Nakajima stratification. In other words, this means that the closure of any $G_{\mathbf{d}^{-}}$-orbit is isomorphic to the quotient of some representation variety of the algebra $\widetilde{\Lambda}_{Q}=B_{Q}$. Our approach allows us to describe this representation variety explicitly. Namely, for a $\mathbf{d}$-dimensional $k Q$ module $M$ recall the $k \widehat{Q}$-module $\widehat{M}$ (used in the construction of desingularizations of quiver Grassmannians). Let $\widehat{\mathbf{d}}$ be the dimension vector of $\widehat{M}$ (note that $\widehat{\mathbf{d}}$ depends on the isomorphism type of $M$, not only on the dimension vector $\mathbf{d}$ ). We prove:

Theorem 1.2. We have the following quotient descriptions of orbit closures:

(i) The closure of the $G_{\mathbf{d}}$-orbit of $M$ is isomorphic to an affine quotient of the variety of $B_{Q}$-representations of dimension vector $\widehat{\mathbf{d}}$.

(ii) The closure of the $G_{\mathbf{d}}$-orbit of $M$ is isomorphic to an affine quotient of

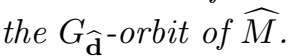

A combinatorial algorithm for computing the dimension vector $\widehat{\mathbf{d}}$ is given in [1] (see also [15], Corollary 3.14, which contains several homological descriptions of $\widehat{\mathbf{d}}$ ). We note that in view of Theorem 1.1, the first part of Theorem 1.2 is proved in [11.

The paper is organized as follows. In Section 2 we collect the definition and main properties of the algebra $B_{Q}$. In Section 3 we describe the quotient maps relating the representation varieties of $\widehat{Q}$ to that of $Q$ and prove Theorem 1.2, In Section 4 the connection with the Hernandez-Leclerc construction is established and Theorem 1.1 is proved.

\section{The algebra $B_{Q}$}

To a Dynkin quiver $Q$ we attach an associative algebra $B_{Q}$ (see [8]). The main ingredients of the construction are a quiver $\widehat{Q}$, an ideal $I \subset k \widehat{Q}$, the algebra $B_{Q}=$ $k \widehat{Q} / I$, and a functor $\Lambda: \bmod k Q \rightarrow \bmod B_{Q} . \quad$ All these objects are introduced and described in 8], except for the ideal $I$, which we describe in Section 4, Let us recall the definitions.

For the path algebra $k Q$ of $Q$, we consider the category mod $k Q$ of finite dimensional left modules over $k Q$, and we consider the subcategory proj $k Q$ of projective modules. We define Hom $(\operatorname{proj} k Q)$ as the $k$-linear category whose objects are morphisms $P \rightarrow Q$ between objects of proj $k Q$ and whose morphism spaces from $P \rightarrow Q$ 
to $R \rightarrow S$ are commutative squares:

$$
\begin{array}{lll}
P & \rightarrow & Q \\
\downarrow & & \downarrow \\
R & \rightarrow & S .
\end{array}
$$

Let $\mathcal{H}_{Q}$ be the full subcategory of $\operatorname{Hom}(\operatorname{proj} k Q)$ with objects being injective morphisms $P \rightarrow Q$ such that the image is not contained in any proper direct summand of $Q$. We define the algebra $B_{Q}$ as $\operatorname{End}(A)^{\text {op }}$ for a minimal additive generator $A$ in $\mathcal{H}_{Q}$. More precisely, the indecomposable objects in $\mathcal{H}_{Q}$ are of two types, namely:

- for every non-projective indecomposable object $U$ of $\bmod k Q$, an embedding $P_{U} \subset Q_{U}$ such that $0 \rightarrow P_{U} \rightarrow Q_{U} \rightarrow U \rightarrow 0$ is a minimal projective resolution,

- for every vertex $i$ of $Q$, the trivial embedding $P_{i}=P_{i}$,

where $P_{i}$ is the projective cover of the simple $k Q$-module $S_{i}$, attached to the vertex $i$.

Thus the algebra $B_{Q}$ is given as

$$
B_{Q}=\operatorname{End}_{\mathcal{H}_{Q}}\left(\bigoplus_{U}\left(P_{U} \subset Q_{U}\right) \oplus \bigoplus_{i}\left(P_{i}=P_{i}\right)\right)^{\mathrm{op}}
$$

Then the quiver $\widehat{Q}$ of $B_{Q}$ is given as follows:

- There are vertices:

- $[U]$ for every non-projective indecomposable in $\bmod k Q$,

- $[i]$ for every vertex of $Q$.

- There are arrows:

$-[U] \rightarrow[V]$ whenever there is an irreducible map $V \rightarrow U$ in $\bmod k Q$,

$-[i] \rightarrow\left[S_{i}\right]$ for every vertex $i$ of $Q$ which is not a sink,

$-\left[\tau^{-1} S_{i}\right] \rightarrow[i]$ for every vertex $i$ of $Q$ which is not a source.

Thus $B_{Q}=k \widehat{Q} / I$ for a certain admissible ideal $I$, and we think of left modules over $B_{Q}$ as certain representations of $\widehat{Q}$. Moreover, the category $\bmod B_{Q}$ of left modules over $B_{Q}$ is equivalent to the category $\bmod \mathcal{H}_{Q}^{\mathrm{op}}$ of contravariant $k$-linear functors from $\mathcal{H}_{Q}$ to the category $\bmod k$ of finite dimensional $k$-vector spaces.

We have a restriction functor res from $\bmod B_{Q}$ to $\bmod k Q$ given on the level of functors as follows: we restrict a functor $F: \mathcal{H}_{Q}^{\mathrm{op}} \rightarrow \bmod k$ to the subcategory of $\mathcal{H}_{Q}$ of objects of the form $(P=P)$ yielding a functor $F:(\operatorname{proj} k Q)^{\text {op }} \rightarrow \bmod k$. The category of such functors is naturally equivalent to $\bmod k Q$.

We give a description of the functor res on the level of representations: given a representation $F$ of $\bmod B_{Q}$, it can be viewed as a special kind of representation of the quiver $\widehat{Q}$. We define the representation $M=\operatorname{res} F$ of $Q$ as follows: first, we define $M_{i}=F_{[i]}$ for all vertices $i$ of $Q$. Let $\alpha: i \rightarrow j$ be an arrow of $Q$. Then $\operatorname{Ext}^{1}\left(S_{i}, S_{j}\right)$ is non-zero (and in fact one-dimensional), thus there exists a non-zero morphism $\tau^{-1} S_{j} \rightarrow S_{i}$ in $\bmod k Q$. Factoring it into irreducibles, there then exists a path $\tau^{-1} S_{j} \rightarrow \ldots \rightarrow S_{i}$ in the Auslander-Reiten quiver of $k Q$. By the definition of $\widehat{Q}$, this yields a path

$$
[i] \rightarrow\left[S_{i}\right] \rightarrow \ldots \rightarrow\left[\tau^{-1} S_{j}\right] \rightarrow[j]
$$

in $\widehat{Q}$. We define $M_{\alpha}: M_{i} \rightarrow M_{j}$ as the composition of the maps in the representation $F$ of $\widehat{Q}$ corresponding to this path. Well-definedness, that is, independence of the path, of this definition follows from the fact that $F$ is a representation of $B_{Q}$, and 
that there is a one-dimensional space of morphisms from $\left(P_{j}=P_{j}\right)$ to $\left(P_{i}=P_{i}\right)$ in $\mathcal{H}_{Q}$.

We define a functor $\Lambda: \bmod k Q \rightarrow \bmod B_{Q}$ as follows: $\Lambda(M)=\widehat{M}$, viewed as a contravariant functor on $\mathcal{H}_{Q}$, is given by

$$
\widehat{M}(P \rightarrow Q)=\operatorname{Im}(\operatorname{Hom}(Q, M) \rightarrow \operatorname{Hom}(P, M)) .
$$

On the level of representations, we can view $\widehat{M}$ as a representation of $\widehat{Q}$ as follows: $\widehat{M}_{[i]}=M_{i}$ and $\widehat{M}_{[U]}=\operatorname{Im}\left(\operatorname{Hom}\left(Q_{U}, M\right) \rightarrow \operatorname{Hom}\left(P_{U}, M\right)\right)$ for a minimal projective resolution $0 \rightarrow P_{U} \rightarrow Q_{U} \rightarrow U \rightarrow 0$ of $U$ in $\bmod Q$.

\section{QUOTIENT MAP}

As before, let $Q$ be a Dynkin quiver with a set of vertices $Q_{0}$ and arrows $\alpha: i \rightarrow j$, and let $k$ be an algebraically closed field of characteristic zero. For a dimension vector $\mathbf{d} \in \mathbf{N} Q_{0}$, we fix vector spaces $M_{i}$ of dimension $d_{i}$ for $i \in Q_{0}$ and define $R_{\mathbf{d}}(Q)=\bigoplus_{\alpha: i \rightarrow j} \operatorname{Hom}_{\bmod k}\left(M_{i}, M_{j}\right)$, on which the group $G_{\mathbf{d}}=\prod_{i \in Q_{0}} \operatorname{GL}\left(M_{i}\right)$ acts via base change $\left(g_{i}\right)_{i}\left(M_{\alpha}\right)_{\alpha}=\left(g_{j} M_{\alpha} g_{i}^{-1}\right)_{\alpha: i \rightarrow j}$. The orbits $\mathcal{O}_{M}$ for this action correspond bijectively to the isomorphism classes $[M]$ of representation of $k Q$ of dimension vector $\mathbf{d}$ by definition. We are interested in the geometry of the Zariski orbit closures $\overline{\mathcal{O}_{M}}$.

We write $\widehat{\mathbf{d}}$ for the dimension vector of $\widehat{M}=\Lambda(M)$ as a representation of $\widehat{Q}$. In particular, $\widehat{\mathbf{d}}$ has entries $\widehat{d}_{U}$ for every non-projective indecomposable $U$ of $k Q$ and entries $\widehat{d}_{i}$ (which coincide with the $d_{i}$ by definition of $\Lambda$ ). We can consider the variety of representations $R_{\widehat{\mathbf{d}}}(\widehat{Q})$, which contains the closed subvariety $R_{\widehat{\mathbf{d}}}\left(B_{Q}\right)$ of representations of $B_{Q}$, that is, those which are annihilated by the ideal $I$.

On $R_{\widehat{\mathrm{d}}}\left(B_{Q}\right)$ we have an action of the structure group $G_{\widehat{\mathrm{d}}}$, which can be canonically written as the product of a group $G_{\widehat{\mathbf{d}}}^{\prime}$ and the group $G_{\mathbf{d}}$, where the first subgroup consists of the structure groups at the vertices $[U]$ of $\widehat{Q}$, and the second subgroup consists of the structure groups at the vertices $[i]$.

The restriction functor res: $\bmod B_{Q} \rightarrow \bmod k Q$ of $[8]$ induces a $G_{\mathbf{d}}$-equivariant map $\pi$ from $R_{\widehat{\mathbf{d}}}\left(B_{Q}\right)$ to $R_{\mathbf{d}}(Q)$.

Proposition 3.1. The image of the induced map $\pi^{*}: k\left[R_{\mathbf{d}}(Q)\right] \rightarrow k\left[R_{\widehat{\mathbf{d}}}\left(B_{Q}\right)\right]$ between coordinate rings coincides with the ring $k\left[R_{\widehat{\mathbf{d}}}\left(B_{Q}\right)\right]^{G_{\widehat{\mathbf{d}}}^{\prime}}$ of $G_{\widehat{\mathbf{d}}}^{\prime}$-invariant functions.

Proof. The following method is the deframing procedure of 9$]$. We introduce an auxilliary quiver $\tilde{Q}$ associated to $\mathbf{d}$ as follows:

- $\tilde{Q}$ has vertices:

- $[U]$ for the non-projective indecomposables $U$ of $\bmod k Q$,

- and one additional vertex $\infty$.

- We have the following arrows in $\tilde{Q}$ :

- arrows $[U] \rightarrow[V]$ corresponding to the irreducible maps $V \rightarrow U$ in $\bmod k Q$,

$-d_{i}$ arrows from $\infty$ to $\left[S_{i}\right]$ for every vertex $i$ of $Q$ which is not a sink,

$-d_{i}$ arrows from $\left[\tau^{-1} S_{i}\right]$ to $\infty$ for every vertex $i$ of $Q$ which is not a source.

We define a dimension vector $\tilde{\mathbf{d}}$ for $\tilde{Q}$ by $\tilde{\mathbf{d}}_{[U]}=\widehat{d}_{[U]}$ and $\tilde{\mathbf{d}}_{\infty}=1$. 
A choice of bases of the spaces $M_{i}$ yields an isomorphism between $R_{\widehat{\mathbf{d}}}(\widehat{Q})$ and $R_{\tilde{\mathbf{d}}}(\tilde{Q})$. Noting that the structure group for the latter variety of representations is isomorphic to $G_{\widehat{\mathbf{d}}}^{\prime} \times \mathbf{G}_{m}$, this isomorphism is also $G_{\widehat{\mathbf{d}}}^{\prime}$-equivariant. In particular, we have

$$
k\left[R_{\widehat{\mathbf{d}}}(\widehat{Q})\right]^{G_{\widehat{\mathbf{d}}}^{\prime}} \simeq k\left[R_{\tilde{\mathbf{d}}}(\tilde{Q})\right]^{G_{\widehat{\mathbf{d}}}^{\prime} \times \mathbf{G}_{m}}=k\left[R_{\tilde{\mathbf{d}}}(\tilde{Q})\right]^{G_{\widehat{\mathbf{d}}}^{\prime}},
$$

since the dilation action at the one-dimension space can be neglected. We now apply the main result of [14 that the latter invariant ring is generated by taking traces along oriented cycles. By cyclic invariance of the trace, noting that the subquiver supported outside the vertex $\infty$ has no oriented cycles, we only need to consider traces along cycles starting and ending in the vertex $\infty$. Translating these invariant functions back to $R_{\widehat{\mathbf{d}}}(\widehat{Q})$, we see that the invariant $\operatorname{ring} k\left[R_{\widehat{\mathbf{d}}}(\widehat{Q})\right]^{G_{\widehat{\mathbf{d}}}^{\prime}}$ is generated by the matrix entries representing paths in $\widehat{Q}$ starting and ending in some of the vertices $[i]$. Without loss of generality, we can restrict to the matrix entries representing paths in $\widehat{Q}$ starting in $[i]$ and ending in $[j]$ whenever we have an arrow $i \rightarrow j$ in $Q$. But the map $\pi$ is induced from the functor res which precisely defines the matrices representing the arrows $i \rightarrow j$ by these paths in $\widehat{Q}$. The proposition is proved.

Remark 3.2. The above argument replaces reference to the theory of Nakajima quiver varieties in [11, Proof of Proposition 9.4] (see also [13, Theorem 1.3).

Theorem 3.3. Via the map $\pi$, the variety $\overline{\mathcal{O}_{M}}$ is the quotient of $\overline{\mathcal{O}_{\widehat{M}}}$ by $G_{\widehat{d}}^{\prime}$.

Proof. By the previous proposition, the map $\pi: R_{\widehat{\mathbf{d}}}\left(B_{Q}\right) \rightarrow \pi\left(R_{\widehat{\mathbf{d}}}\left(B_{Q}\right)\right) \subset R_{\mathbf{d}}(Q)$ is a quotient by $G_{\widehat{\mathbf{d}}}^{\prime}$. The subset $\overline{\mathcal{O}_{\widehat{M}}}$ is closed and $G_{\widehat{\mathbf{d}}}^{\prime}$-invariant, thus the restriction $\pi: \overline{\mathcal{O}_{\widehat{M}}} \rightarrow \pi\left(\overline{\mathcal{O}_{\widehat{M}}}\right)$ is a quotient. The latter image is closed by properties of quotients, it is $G_{\mathbf{d}^{-}}$-stable by $G_{\mathbf{d}}$-equivariance of $\pi$, and it contains $\pi\left(\mathcal{O}_{\widehat{M}}\right)=\mathcal{O}_{M}$ as a dense subset. Thus it coincides with $\overline{\mathcal{O}_{M}}$. Again by properties of quotients, the restriction is a quotient itself.

We will show that, in fact, the whole representation variety $R_{\widehat{\mathbf{d}}}\left(B_{Q}\right)$ is mapped to the closure of $\mathcal{O}_{M}$ under the map $\pi$. To do this, we have to know the precise relation between the functors $F$ and $\widehat{\operatorname{res} F}$, which we describe using the methods of [8]:

Theorem 3.4. For every functor $F$ in $\bmod \mathcal{H}_{Q}^{\mathrm{op}}$, there exist canonical exact sequences

$$
\begin{gathered}
0 \rightarrow F_{1} \rightarrow F \rightarrow \operatorname{Ext}_{B_{Q}}^{1}\left(\widehat{\text { Coker }_{-}}, F\right) \rightarrow 0, \\
0 \rightarrow \operatorname{Ext}_{B_{Q}}^{1}\left(F_{1}, \tau_{Q} \widehat{\text { Coker }_{-}}\right)^{*} \rightarrow F_{1} \rightarrow \widehat{\operatorname{res} F} \rightarrow 0,
\end{gathered}
$$

and dually

$$
\begin{aligned}
& 0 \rightarrow \operatorname{Ext}_{B_{Q}}^{1}\left(F, \tau_{Q} \widehat{\text { Coker }_{-}}\right)^{*} \rightarrow F \rightarrow F_{2} \rightarrow 0, \\
& 0 \rightarrow \widehat{\operatorname{res} F} \rightarrow F_{2} \rightarrow \operatorname{Ext}_{B_{Q}}^{1}\left(\widehat{\text { Coker }}, F_{2}\right) \rightarrow 0 .
\end{aligned}
$$

Proof. For every functor $F$, we define functors $F_{1}, F_{2}$ and $F_{3}$ as follows: given an object $P \subset Q$ of $\mathcal{H}_{Q}$, we have a canonical sequence of maps

$$
(P=P) \stackrel{f}{\longrightarrow}(P \subset Q) \stackrel{g}{\longrightarrow}(Q=Q)
$$


in $\mathcal{H}_{Q}$ by [8, Proof of Lemma 5.3]. We define

$$
\begin{aligned}
& F_{1}(P \subset Q)=\operatorname{Im}(F(Q=Q) \stackrel{F(g)}{\longrightarrow} F(P \subset Q)), \\
& F_{2}(P \subset Q)=\operatorname{Im}(F(P \subset Q) \stackrel{F(f)}{\longrightarrow} F(P=P)), \\
& F_{3}(P \subset Q)=\operatorname{Im}(F(Q=Q) \stackrel{F(g \circ f)}{\longrightarrow} F(P=P)),
\end{aligned}
$$

with the natural definition on morphisms. In particular, $F_{i}(P=P)=F(P=P)$, for every $i=1,2,3$. We thus have

$$
F_{3}=\left(F_{1}\right)_{2}=\left(F_{2}\right)_{1}=\widehat{\operatorname{res} F}
$$

by definition. Thus the existence of the second and fourth claimed exact sequence follows from existence of the first and third. Existence of the first sequence is equivalent to exactness of

$$
0 \rightarrow \operatorname{Im}(F(Q=Q) \rightarrow F(P \subset Q)) \rightarrow F(P \subset Q) \rightarrow \operatorname{Ext}^{1}(\widehat{Q / P}, F) \rightarrow 0
$$

for all objects $P \subset Q$ of $\mathcal{H}_{Q}$. By [8, Proof of Theorem 5.6], we have a projective resolution of functors

$$
0 \rightarrow \operatorname{Hom}\left({ }_{-},(P \subset Q)\right) \rightarrow \operatorname{Hom}\left({ }_{-},(Q=Q)\right) \rightarrow \widehat{Q / P} \rightarrow 0 .
$$

Applying $\operatorname{Hom}(, F)$ to this sequence and using Yoneda's Lemma, this yields a right exact sequence

$$
F(Q=Q) \rightarrow F(P \subset Q) \rightarrow \operatorname{Ext}^{1}(\widehat{Q / P}, F) \rightarrow 0 .
$$

This proves the above exactness claim.

To prove the existence of the third exact sequence, we use methods of AuslanderReiten theory, to which we refer to [2]. We denote by $\tau=\tau_{Q}$ the Auslander-Reiten translate in the category $\bmod k Q$, and by $\tau_{B_{Q}}$ the Auslander-Reiten translate in the category $\bmod B_{Q}$.

As above, we have to prove exactness of

$$
\left.0 \rightarrow \operatorname{Ext}^{1}(F, \widehat{\tau(Q / P})\right)^{*} \rightarrow F(P \subset Q) \rightarrow \operatorname{Im}(F(P \subset Q) \rightarrow F(P=P)) \rightarrow 0 .
$$

Since the image of $\Lambda$ consists of objects of injective dimension at most one 8 , Theorem 5.6], we can apply the Auslander-Reiten formula [2, Corollary 2.15] and identify

$$
\left.\left.\operatorname{Ext}^{1}(F, \tau \widehat{(Q / P})\right)^{*} \simeq \operatorname{Hom}\left(\tau_{B_{Q}}^{-1} \tau \widehat{(Q / P}\right), F\right) .
$$

We use the (inverse) Nakayama functors $\nu^{(-)}$(resp. $\nu_{B_{Q}}^{(-)}$) of the category mod $k Q$ (resp. $\left.\bmod B_{Q}\right)$; see [2, IV.2]. The functor $\nu_{B_{Q}}^{-}$is given, by definition, by $\nu_{B_{Q}}^{-}\left(\operatorname{Hom}\left((P \subset Q),{ }_{-}\right)^{*}\right)=\operatorname{Hom}\left({ }_{-},(P \subset Q)\right)$. Starting from an object $P \subset Q$ of $\mathcal{H}_{Q}$, we consider the exact sequence $0 \rightarrow P \rightarrow Q \rightarrow Q / P \rightarrow 0$, which induces an exact sequence

$$
0 \rightarrow \tau(Q / P) \rightarrow \nu P \rightarrow \nu Q \rightarrow \nu(Q / P)=0
$$

by [2, Proposition 2.4.(a)]; the last equality follows since $Q / P$ has no projective direct summand by assumption. By [8, Proof of Theorem 5.6], this injective coresolution of $\tau(Q / P)$ induces an injective coresolution

$$
0 \rightarrow \tau \widehat{(Q / P}) \rightarrow \operatorname{Hom}\left((P=P),{ }_{-}\right)^{*} \rightarrow \operatorname{Hom}\left((P \subset Q),{ }_{-}\right)^{*} \rightarrow 0
$$


of $\tau \widehat{(Q / P)}$. This in turn, by [2, Proposition 2.4.(b)], yields a right exact sequence

$$
\underbrace{\nu_{B_{Q}}^{-} \operatorname{Hom}\left((P=P),{ }_{-}\right)^{*}}_{\simeq \operatorname{Hom}(-,(P=P))} \rightarrow \underbrace{\nu_{B_{Q}}^{-} \operatorname{Hom}\left((P \subset Q),{ }_{-}\right)^{*}}_{\simeq \operatorname{Hom}(-,(P \subset Q))} \rightarrow \tau_{B_{Q}}^{-1} \widehat{\tau Q / P} \rightarrow 0 .
$$

Applying $\operatorname{Hom}(-, F)$ to this sequence and using Yoneda's Lemma, this yields a sequence

$$
0 \rightarrow \operatorname{Hom}\left(\tau_{B_{Q}}^{-1} \widehat{Q / P}, F\right) \rightarrow F(P \subset Q) \rightarrow F(P=P) .
$$

The above claimed exactness follows. The theorem is proved.

Corollary 3.5. The following conditions are equivalent for a functor $F$ in mod $\mathcal{H}_{Q}^{\mathrm{op}}$ and a representation $N$ of $\bmod k Q$ :

(i) $\operatorname{res} F \simeq N$,

(ii) There exist exact sequences

$$
0 \rightarrow G \rightarrow F \rightarrow F^{\prime} \rightarrow 0 \text { and } 0 \rightarrow F^{\prime \prime} \rightarrow G \rightarrow \widehat{N} \rightarrow 0
$$

such that $\operatorname{res} F^{\prime}=0=\operatorname{res} F^{\prime \prime}$.

Moreover, a functor $F$ belongs to the essential image of $\Lambda$ if and only if $\operatorname{Ext}^{1}(F, \widehat{V})=$ $0=\operatorname{Ext}^{1}(\widehat{V}, F)$ for all $V$.

Proof. The first claim follows from the previous theorem and exactness of the functor res. The second claim follows from the previous theorem and 8, Theorem $5.6]$.

Remark 3.6. The second statement of the corollary gives an alternative description of the essential image of the functor $\Lambda$ to [8, Proposition 6.11].

These homological properties allow us to derive the following information on the quotient map $\pi$ and the structure of its fibres:

Corollary 3.7. The quotient map $\pi$ maps the whole variety $R_{\widehat{\mathbf{d}}}\left(B_{Q}\right)$ onto $\overline{\mathcal{O}_{M}}$.

Proof. Given a functor $F$ of the same dimension vector as $\widehat{M}$ with restriction $N=\operatorname{res} F$, we thus have to prove that $\mathcal{O}_{N}$ belongs to the closure of $\mathcal{O}_{M}$. By [6], the latter is equivalent to $\operatorname{dim} \operatorname{Hom}(U, M) \leq \operatorname{dim} \operatorname{Hom}(U, N)$ holding for all non-projective indecomposables $U$. For each such $U$, use a minimal projective resolution $0 \rightarrow P \rightarrow Q \rightarrow U \rightarrow 0$ as before and calculate using the definition of the functor $\Lambda$ :

$$
\begin{aligned}
\operatorname{dim}_{[U]} F= & \operatorname{dim}_{[U]} \widehat{M}=\operatorname{dim} \operatorname{Im}(\operatorname{Hom}(Q, M) \rightarrow \operatorname{Hom}(P, M)) \\
& =\operatorname{dim} \operatorname{Hom}(Q, M)-\operatorname{dim} \operatorname{Hom}(U, M) .
\end{aligned}
$$

By the previous theorem, we have $\operatorname{dim}_{[U]} F \geq \operatorname{dim}_{[U]} \widehat{N}$, and thus

$$
\begin{aligned}
& \operatorname{dim} \operatorname{Hom}(U, M)=\operatorname{dim} \operatorname{Hom}(Q, M)-\operatorname{dim}_{[U]} F \\
\leq & \operatorname{dim} \operatorname{Hom}(Q, N)-\operatorname{dim}_{[U]} \widehat{N}=\operatorname{dim} \operatorname{Hom}(U, N),
\end{aligned}
$$

proving the claim.

Remark 3.8. If $\widehat{M}$ and $\widehat{N}$ have the same dimension vector, then $M \simeq N$. Indeed, by the lemma above we have $N \in \overline{\mathcal{O}_{M}}$ and $M \in \overline{\mathcal{O}_{N}}$. 
One can ask whether this description of the orbit closure $\overline{\mathcal{O}_{M}}$ as an affine quotient has applications to the study of its geometric properties, in the spirit of the proof [1] - in the case of $Q$ being an equioriented type $A$ quiver - of such orbit closures being normal Cohen-Macaulay varieties with at most rational singularities. To this effect, we consider the stratification of the representation variety $R_{\widehat{\mathbf{d}}}\left(B_{Q}\right)$ by the inverse images $\pi^{-1}\left(\mathcal{O}_{N}\right)$ of the orbits in $\overline{\mathcal{O}_{M}}$ under the quotient map $\pi$ and formulate the following:

Conjecture 3.9. For every $N$, we have $\operatorname{dim} \pi^{-1}\left(\mathcal{O}_{N}\right) \leq \operatorname{dim} \overline{\mathcal{O}_{\overparen{M}}}$, with equality holding only for $N=M$.

We discuss briefly the potential applications of a positive answer to this conjecture. Using the arguments of [3, 2.1,2.2], the homological Euler form of the algebra $B_{Q}$ (being of global dimension at most two) can be calculated to give $\langle\widehat{\mathbf{d}}, \widehat{\mathbf{d}}\rangle=\operatorname{dim} \operatorname{End}(M)$ if $\widehat{\mathbf{d}}=\operatorname{dim} \widehat{M}$. It follows that $\overline{\mathcal{O}_{\widehat{M}}}$ is an irreducible component of $R_{\widehat{\mathbf{d}}}\left(B_{Q}\right)$, and that every irreducible component has at least this dimension. Assuming the above conjecture, $\overline{\mathcal{O}_{\widehat{M}}}=R_{\widehat{\mathbf{d}}}\left(B_{Q}\right)$ is the only irreducible component, which is locally a complete intersection and thus Cohen-Macaulay. A more refined analysis of the strata $\pi^{-1}\left(\mathcal{O}_{N}\right)$ is expected to prove regularity in codimension one of $R_{\widehat{\mathbf{d}}}\left(B_{Q}\right)$. This implies normality, and could thus provide a uniform proof of normality of every orbit closure $\overline{\mathcal{O}_{M}}$. Note that the first statement of Corollary 3.5 gives an intrinsic description of the stratum $\pi^{-1}\left(\mathcal{O}_{N}\right)$.

\section{The Hernandez-LeClerc COnstruction And GRADED NAKAJIMA VARIETIES}

In [11, Section 9.3] an algebra $\tilde{\Lambda}_{Q}$ is introduced. Let us collect the main ingredients of the Hernandez-Leclerc construction. Let $Q$ be a Dynkin quiver with the set of vertices $I$ of cardinality $n$ and let $\mathfrak{g}$ be the corresponding simple Lie algebra. We denote by $\alpha_{i}, i \in I$ simple roots, by $\triangle_{+}$and $\triangle_{-}$the sets of positive and negative roots, and by $W$ the Weyl group of $\mathfrak{g}$. Let $s_{i} \in W, i \in I$ be the simple reflections, $s_{i}(\lambda)=\lambda-(\lambda, \alpha) \alpha_{i}$. A Coxeter element $C \in W$ is the product $s_{i_{1}} s_{i_{2}} \ldots s_{i_{n}}$ of simple reflections each showing up exactly once. For a quiver $Q$ we denote by $s_{i} Q$ a new quiver, which is obtained from $Q$ by reversing all arrows at the vertex $i$. The Coxeter element $C$ is said to be adapted to $Q$, if $i_{1}$ is a source of $Q, i_{2}$ is a source of $s_{i_{1}} Q, i_{k+1}$ is a source of $s_{i_{1}} \ldots s_{i_{k}} Q$. For example, if $Q=1 \rightarrow 2 \leftarrow 3 \rightarrow 4$, then $C=s_{1} s_{3} s_{2} s_{4}$.

A height function $\xi: I \rightarrow \mathbb{Z}$ is a function satisfying $\xi_{j}=\xi_{i}-1$ if there is an arrow $i \rightarrow j$ in $Q$. For $Q=1 \rightarrow 2 \leftarrow 3 \rightarrow 4$ a possible height function is $\xi_{4}=1$, $\xi_{3}=2, \xi_{2}=1, \xi_{1}=2$. Let us define a set

$$
\widehat{I}=\left\{(i, p) \in I \times \mathbb{Z}: p-\xi_{i} \in 2 \mathbb{Z}\right\} .
$$

A crucial role in the whole picture is played by the following bijection $\varphi: \widehat{I} \rightarrow$ $\triangle_{+} \times \mathbb{Z}$. The bijection $\varphi$ is defined as follows. First, for a vertex $i \in I$ we denote by $\gamma_{i} \in \triangle_{+}$the sum of all simple roots $\alpha_{j}$ such that there is a path form $j$ to $i$ in $Q$ (these are exactly the vertices showing up in the injective envelope of the simple module attached to the vertex $i$ ). Now $\varphi$ is defined by the rules:

- $\varphi\left(i, \xi_{i}\right)=\left(\gamma_{i}, 0\right)$;

- Let $\varphi(i, p)=(\beta, m)$. Then $\varphi(i, p-2)=\left\{\begin{array}{l}(C(\beta), m), \text { if } C(\beta) \in \triangle_{+}, \\ (-C(\beta), m-1), \text { if } C(\beta) \in \triangle_{-} .\end{array}\right.$ 
Remark 4.1. It follows that if $\varphi(i, p)=(\beta, m)$, then

$$
\varphi(i, p+2)=\left\{\begin{array}{l}
\left(C^{-1}(\beta), m\right), \text { if } C^{-1}(\beta) \in \triangle_{+}, \\
\left(-C^{-1}(\beta), m+1\right), \text { if } C^{-1}(\beta) \in \triangle_{-} .
\end{array}\right.
$$

Example 4.2. Let us give an example. Let $Q=1 \rightarrow 2 \rightarrow 3 \rightarrow 4$ and the height function is fixed as $\xi_{1}=4, \xi_{2}=3, \xi_{3}=2, \xi_{4}=1$. The underlying Lie algebra is $\mathfrak{s l}_{5}$ and $\triangle_{+}$consists of the roots

$$
\alpha_{i, j}=\alpha_{i}+\alpha_{i+1}+\cdots+\alpha_{j}, 1 \leq i \leq j \leq 4 .
$$

The adapted Coxeter element $C$ is equal to $s_{1} s_{2} s_{3} s_{4}$. Let us draw the set $\widehat{I}$ as follows:

$\begin{array}{llll}(1,6) & & (3,6) & \\ & (2,5) & & (4,5) \\ (1,4) & & (3,4) & \\ & (2,3) & & (4,3) \\ (1,2) & & (3,2) & \\ & (2,1) & & (4,1) \\ (1,0) & & (3,0) & \\ (1,-2) & (2,-1) & & (4,-1) \\ (1,-4) & & (3,-2) & \\ & & (3,-4) & \end{array}$

Then the corresponding images $\varphi(i, p)$ look as follows:

$$
\begin{aligned}
& \left(\alpha_{1,4}, 1\right) \quad\left(\alpha_{2,3}, 1\right) \\
& \left(\alpha_{1,1}, 0\right) \quad\left(\alpha_{2,4}, 1\right) \quad\left(\alpha_{3,4}, 1\right) \quad\left(\alpha_{3,3}, 1\right) \\
& \begin{array}{llll}
\left(\alpha_{2,2}, 0\right) & \left(\alpha_{1,2}, 0\right) & & \left(\alpha_{4,4}, 1\right) \\
& \left(\alpha_{2,3}, 0\right) & \left(\alpha_{1,3}, 0\right) & \left(\alpha_{1,4}, 0\right)
\end{array} \\
& \begin{array}{lll}
\left(\alpha_{3,3}, 0\right) & \left(\alpha_{2,4}, 0\right) & \left(\alpha_{3,4}, 0\right)
\end{array} \\
& \begin{array}{lll}
\left(\alpha_{4,4}, 0\right) & \left(\alpha_{1,2},-1\right) & \left(\alpha_{1,3},-1\right)
\end{array} \\
& \left(\alpha_{1,4},-1\right) \quad\left(\alpha_{2,3},-1\right)
\end{aligned}
$$

In [1] the authors define the following graph $\widetilde{\Gamma}_{Q}$. The vertices of $\widetilde{\Gamma}_{Q}$ are of two sorts:

$$
w_{j}(p) \text {, where } \varphi(j, p)=\left(\alpha_{i}, 0\right) \text { for some } i \in I
$$

and

$$
v_{j}(p-1) \text {, where } \varphi(j, p) \in \triangle_{+} \times\{0\} \text { and } \varphi(j, p-2) \in \triangle_{+} \times\{0\} .
$$

In particular, the number of vertices $w_{j}(p)$ is equal to the number of simple roots (the number of vertices of $Q$ ) and (as we will see) the number of vertices $v_{j}(p-1)$ is equal to the number of positive roots minus the number of simple roots. In the example above ( $Q$ equioriented type $A_{4}$ ) the vertices are

$$
w_{1}(4), w_{1}(2), w_{1}(0), w_{1}(-2), v_{1}(3), v_{1}(1), v_{1}(-1), v_{2}(2), v_{2}(0), v_{3}(1) .
$$


Remark 4.3. Recall that our $\widehat{Q}$ from [8] also has two sorts of vertices: vertices $[i]$, $i \in I$ and vertices $[U]$, that correspond to indecomposable non-projective $U$. In what follows we denote the indecomposable representation of $k Q$ corresponding to a positive root $\beta$ by $U_{\beta}$. Also for an indecomposable representation $U$ we denote by $\operatorname{dim} U \in \triangle_{+}$the $\operatorname{root} \sum \operatorname{dim} U_{i} \alpha_{i}$.

Lemma 4.4. Let $\varphi(j, p)=(\beta, 0) \in \triangle_{+} \times\{0\}$. Then $\varphi(j, p-2) \in \triangle_{+} \times\{0\}$ if and only if $U_{\beta}$ is non-projective.

Proof. The conditions $\varphi(j, p)=(\beta, 0) \in \triangle_{+} \times\{0\}$ and $\varphi(j, p-2) \notin \triangle_{+} \times\{0\}$ mean that $C(\beta) \in \triangle_{-}$. Hence we are looking for positive roots $\beta$ such that $C$ maps them to a negative root. We want to show that these roots correspond to the ones defining projective indecomposable modules. This is proved in Proposition 4.1 of [12] (the dual version of that, to be precise).

Recall (see [1]) that the arrows of $\widetilde{\Gamma}_{Q}$ are of three types:

- $a_{j}(p): w_{j}(p) \rightarrow v_{j}(p-1)$,

- $b_{j}(p): v_{j}(p) \rightarrow w_{j}(p-1)$,

- $B_{i j}(p): v_{i}(p) \rightarrow v_{j}(p-1)$ if there is an arrow $i \rightarrow j$ or $j \rightarrow i$.

Remark 4.5. In $\widehat{Q}$ we also have three types of arrows.

Example 4.6. Let $Q=1 \rightarrow 2 \rightarrow 3 \rightarrow 4$ and $\xi_{1}=4, \xi_{2}=3, \xi_{3}=2, \xi_{4}=1$ as above. Then the quiver $\widetilde{\Gamma}_{Q}$ looks as follows:

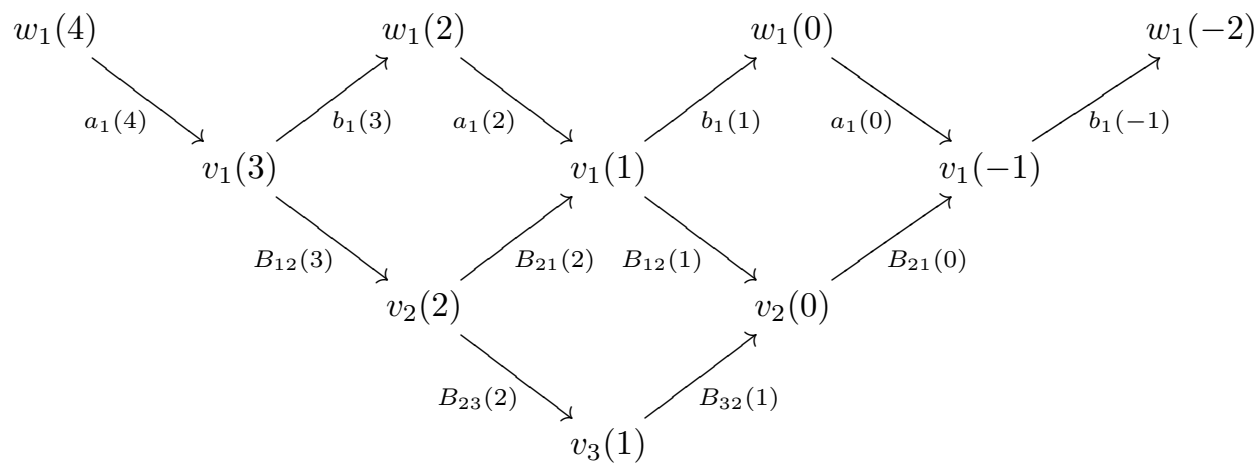

Example 4.7. Let $Q=1 \rightarrow 2 \leftarrow 3$ and $\xi_{1}=2, \xi_{2}=1, \xi_{3}=2$. Then $C=s_{1} s_{3} s_{2}$ and the bijection $\varphi: \widehat{I} \rightarrow \triangle_{+} \times \mathbb{Z}$ looks as follows:

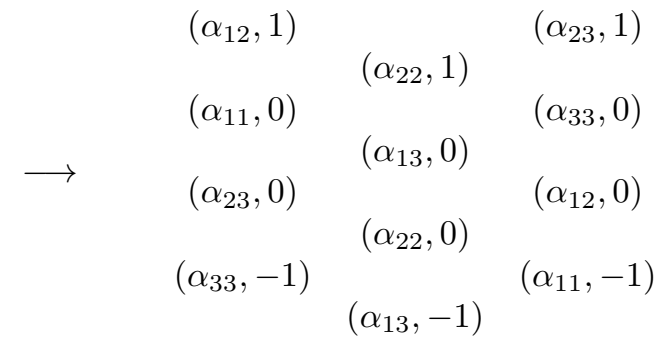


Then we have vertices $w_{1}(2), w_{2}(-1), w_{3}(2), v_{1}(1), v_{2}(0), v_{3}(1)$ and the quiver $\widetilde{\Gamma}_{Q}$ looks as follows:

$$
w_{1}(2) \stackrel{a_{1}(2)}{\longrightarrow} v_{1}(1) \stackrel{B_{21}(1)}{\longrightarrow} v_{2}(0) \stackrel{B_{32}(1)}{\longleftarrow} v_{3}(1) \stackrel{a_{3}(2)}{\longleftarrow} w_{3}(2)
$$

This picture agrees with the corresponding example in 8 .

Lemma 4.8. (a) Let $\varphi(j, p)=(\beta, 0)$ and $\varphi(j, p-2)=(\gamma, 0), \beta, \gamma \in \triangle_{+}$. Then $\tau U_{\beta}=U_{\gamma}$.

(b) Assume that the vertices $i, j \in I$ are connected by an arrow $i \rightarrow j$. Let $\varphi(i, p)=(\beta, 0)$ and $\varphi(j, p-1)=(\gamma, 0)$ with $\beta$ and $\gamma$ in $\triangle_{+}$. Then there is an irreducible map $U_{\gamma} \rightarrow U_{\beta}$.

Proof. By definition $\gamma=C \beta=C \operatorname{dim} U_{\beta}=\operatorname{dim} \tau U_{\beta}$ (for the last equality see e.g. [16, 2.4 (4)] or [2, lemma 5.8]). Since the indecomposable $Q$-representations, for $\mathrm{Q}$ Dynkin, are uniquely determined by their dimension vector, part (a) follows.

To prove part (b) we notice that for $p=\xi_{i}, \varphi\left(i, \xi_{i}\right)=\left(\operatorname{dim} I_{i}, 0\right)$ by definition of $\varphi$; and in view of part (a), $U_{\beta}=\tau^{k} I_{i}$ for $k$ given by $2 k=\xi_{i}-p$. Similarly $U_{\gamma}=\tau^{l} I_{j}$ with $2 l=\xi_{j}-p+1$. Since there is an arrow $i \rightarrow j$ in $Q$, then $\xi_{j}=\xi_{i}-1$ by definition of $\xi$, and hence $k=l$. Again by our assumption that there is an arrow $i \rightarrow j, I_{i}$ is an indecomposable direct summand of $I_{j} / \operatorname{soc} \mathrm{I}_{\mathrm{j}}$, and hence there is an irreducible map $I_{j} \rightarrow I_{i}$ (see e.g. [2, remark IV.4.3 (b)]). It follows that there is an irreducible morphism $\tau^{s} I_{j} \rightarrow \tau^{s} I_{i}$ for every $s$, by functoriality of $\tau$, and hence also for $s=k=l$.

Proposition 4.9. The quivers $\widetilde{\Gamma}_{Q}$ from [1] and $\widehat{Q}$ from [8] are isomorphic. More precisely, there exists an isomorphism between $\widetilde{\Gamma}_{Q}$ and $\widehat{Q}$ sending $w_{j}(p)$ to $[i]$, if $\varphi(j, p)=\left(\alpha_{i}, 0\right)$, and sending $v_{j}(p-1)$ to $\left[U_{\beta}\right]$, if $\varphi(j, p)=(\beta, 0)$.

Proof. We need to show that the arrows $a_{j}(p), b_{j}(p)$ and $B_{i j}(p)$ are in correspondence with the arrows in $\widehat{Q}$.

First, let us look at $a_{j}(p): w_{j}(p) \rightarrow v_{j}(p-1)$. We know that $\varphi(j, p)=\left(\alpha_{i}, 0\right)$ (from the definition of $\left.w_{j}(p)\right)$ and hence $v_{j}(p-1)$ corresponds to $U_{\alpha_{i}}$ (the simple representation attached to the vertex $i$ ). We thus get an arrow $[i] \rightarrow\left[U_{\alpha_{i}}\right]$, which is indeed present in $\widehat{Q}$.

Second, let us look at $b_{j}(p): v_{j}(p) \rightarrow w_{j}(p-1)$. We know that $\varphi(j, p-1)=$ $\left(\alpha_{i}, 0\right)$. The vertex $v_{j}(p)$ corresponds to the pair $(j, p+1) \in \widehat{I}$. Let $\varphi(j, p+1)=\beta$. From Lemma 4.8, part (a), we know that $U_{\beta}=\tau^{-1} U_{\alpha_{i}}$. We thus obtain an arrow $\left[\tau^{-1} U_{\alpha_{i}}\right] \rightarrow[i]$, which is indeed present in $\widehat{Q}$.

Finally we consider the arrows $B_{i, j}(p): v_{i}(p) \rightarrow v_{j}(p-1)$, where $i$ and $j$ are connected by an arrow $(i \rightarrow j$ or $j \rightarrow i$ ). The existence of the corresponding arrows in $\widehat{Q}$ follows from Lemma 4.8, part (b).

Finally, the algebra $\tilde{\Lambda}_{Q}$ is defined in [11, Section 9.3] as the path algebra of the quiver $\widetilde{\Gamma}_{Q}$ subject to the relations

$$
a_{i}(p-1) b_{i}(p)=\sum_{j-i} \epsilon(i, j) B_{j i}(p-1) B_{i j}(p),
$$


for all vertices $i$ and all $p$, where the sum ranges over all vertices $j$ adjacent to $i$, and $\epsilon(i, j)$ is an appropriate sign. For our purposes, a modified form of these relations (without the signs) will be more suitable:

Lemma 4.10. The algebra $\widetilde{\Lambda}_{Q}$ is isomorphic to the path algebra of the quiver $\widetilde{\Gamma}_{Q}$ subject to the relations

$$
a_{i}(p-1) b_{i}(p)=\sum_{j-i} B_{j i}(p-1) B_{i j}(p),
$$

for all vertices $i$ and all $p$, where the sum ranges over all vertices $j$ adjacent to $i$.

Proof. We twist the arrow $B_{i, j}(p)$ by a $\operatorname{sign} \epsilon(i, j, p)$. Then the $\operatorname{sign} \epsilon(i, j)$ in (4.1) vanishes and gives the desired relation (4.2) if and only if $\epsilon(j, i, p-1) \epsilon(i, j, p) \epsilon(i, j)=$ 1 for all $i, j, p$, which can be rewritten as

$$
\epsilon(j, i, p-1)=\epsilon(i, j) \epsilon(i, j, p) .
$$

This gives a way to define the correct signs inductively: for every pair of adjacent vertices $i, j$ in $Q$, we take the maximal $p$ such that $B_{i, j}(p)$ exists and choose $\epsilon(i, j, p)$ arbitrarily. Then for all smaller $q$, the above relation tells us how to define the sign $\epsilon(i, j, q)$.

Theorem 4.11. The algebra $\tilde{\Lambda}_{Q}$ is isomorphic to the algebra $B_{Q}$.

Proof. We compute defining relations for the algebra $B_{Q}$. Since $B_{Q}$ is of global dimension at most two, we can localize the relations by computing $\mathrm{Ext}^{2}$ between simple objects by [3, Section 1], which in the language of contravariant functors on $\mathcal{H}_{Q}$ are the $S_{P_{U} \subset Q_{U}}$ for $U$ a non-projective indecomposable over $k Q$, and the $S_{P_{i}=P_{i}}$ for the vertices $i$ of $Q$. It is shown in [8] that the $S_{P_{i}=P_{i}}$ have projective and injective dimension at most one, thus it suffices to compute $\operatorname{Ext}^{2}\left(S_{P_{U} \subset Q_{U}}, S_{P_{V} \subset Q_{V}}\right)$ for two non-projective indecomposables $U$ and $V$ over $k Q$. Moreover, it is shown in [8] that $\operatorname{Ext}^{*}\left(S_{P_{U} \subset Q_{U}}, F\right)$ can be computed as the homology of the complex

$$
F\left(P_{U} \subset Q_{U}\right) \rightarrow F\left(P_{B} \subset Q_{B}\right) \rightarrow F\left(P_{\tau U} \subset Q_{\tau U}\right),
$$

where $0 \rightarrow \tau U \rightarrow B \rightarrow U \rightarrow 0$ is the Auslander-Reiten sequence ending in $U$, and $P_{B}=P_{U} \oplus P_{\tau U}, Q_{B}=Q_{U} \oplus Q_{\tau U}$ (note that $P_{B}$ and $Q_{B}$ do not necessarily define a minimal resolution of $B$ ). Thus, if $\operatorname{Ext}^{2}\left(S_{P_{U} \subset Q_{U}}, S_{P_{V} \subset Q_{V}}\right)$ is non-zero, we have $V=\tau U$, in which case $\operatorname{Ext}^{2}\left(S_{P_{U} \subset Q_{U}}, S_{P_{V} \subset Q_{V}}\right)$ is one-dimensional. Thus to define the algebra $B_{Q}$ as a quotient of $k \widehat{Q}$, it suffices to determine a single relation involving paths from the vertex $[U]$ to the vertex $[\tau U]$. To do this, we compare morphisms in $\mathcal{H}_{Q}$ and in $\bmod k Q$. By [8], we have an isomorphism

$$
\underline{\operatorname{Hom}}_{\mathcal{H}_{Q}}((P \subset Q),(R \subset S)) \simeq \operatorname{Hom}_{k Q}(Q / P, S / R),
$$

where $\underline{\operatorname{Hom}}_{\mathcal{H}_{Q}}((P \subset Q),(R \subset S))$ is defined as the quotient of $\operatorname{Hom}_{\mathcal{H}_{Q}}((P \subset$ $Q),(R \subset S))$ by the subspace generated by morphisms factoring through an object of $\mathcal{H}_{Q}$ of the form $(T=T)$. In particular, we have $\operatorname{Hom}_{\mathcal{H}_{Q}}\left(P_{U} \subset Q_{U}, P_{V} \subset Q_{V}\right) \simeq$ $\operatorname{Hom}(U, V)$ if there exists an irreducible map from $U$ to $V$, and $\operatorname{Hom}_{\mathcal{H}_{Q}}\left(P_{\tau U} \subset\right.$ $\left.Q_{\tau U}, P_{U} \subset Q_{U}\right) \simeq \operatorname{Hom}(\tau U, U)$ if there is no vertex $[j]$ between $[\tau U]$ and $[U]$ in $\widehat{Q}$. First let us assume that this is the case. Then the relation in $B_{Q}$ between $[\tau U]$ and $[U]$ is the mesh relation, that is, the sum over all paths from $[\tau U]$ to $[U]$ is zero. Otherwise, let us assume that there is a vertex $[j]$ between $[\tau U]$ and $[U]$. Then, by definition of $\widehat{Q}$, we have $U=S_{i}$ and $\tau U=\tau S_{i}=S_{j}$ for vertices $i$ and $j$ of $Q$. 
In particular, we have an Auslander-Reiten sequence $0 \rightarrow S_{j} \rightarrow B \rightarrow S_{i} \rightarrow 0$ in $\bmod k Q$, so that there exists an arrow $i \rightarrow j$ in $Q$ and $B$ is the two-dimensional indecomposable supported on $i$ and $j$. A direct computation using the projective resolution $0 \rightarrow \bigoplus_{j \rightarrow k} P_{k} \rightarrow P_{j} \rightarrow S_{j} \rightarrow 0$ of $S_{j}$ (and similarly for $S_{i}$ ) shows that in this case we have a commutativity relation for the commutative square

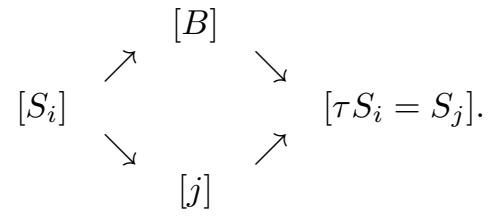

We conclude that the defining relations of $B_{Q}$ coincide with the defining relations (4.2) of $\tilde{\Lambda}_{Q}$ under the identification between the quivers $\widetilde{\Gamma}_{Q}$ and $\widehat{Q}$.

\section{ACKNOWLEGMENTS}

The authors would like to thank K. Bongartz, B. Leclerc, B. Keller and S. Scherotzke for helpful discussions.

The work of Evgeny Feigin was partially supported by the Russian President Grant MK-3312.2012.1, by the Dynasty Foundation, by the AG Laboratory HSE, RF government grant, ag. 11.G34.31.0023, by the RFBR grants 12-01-00070, 12-01-00944, 12-01-33101, 13-01-12401/13 and by the Russian Ministry of Education and Science under the grant 2012-1.1-12-000-1011-016. This study comprises research fundings from the "Representation Theory in Geometry and in Mathematical Physics carried out within The National Research University Higher School of Economics" Academic Fund Program in 2012, grant no. 12-05-0014. This study was carried out within the National Research University Higher School of Economics Academic Fund Program in 2012-2013, research grant no. 11-01-0017.

The work of Giovanni Cerulli Irelli was supported by DFG grant SPP 1388.

\section{REFERENCES}

[1] S. Abeasis, A. Del Fra, and H. Kraft, The geometry of representations of $A_{m}$, Math. Ann. 256 (1981), no. 3, 401-418, DOI 10.1007/BF01679706. MR626958 (83h:14038)

[2] Ibrahim Assem, Daniel Simson, and Andrzej Skowroński, Elements of the representation theory of associative algebras. Vol. 1, Techniques of representation theory. London Mathematical Society Student Texts, vol. 65, Cambridge University Press, Cambridge, 2006. MR2197389 (2006j:16020)

[3] Klaus Bongartz, Algebras and quadratic forms, J. London Math. Soc. (2) 28 (1983), no. 3, 461-469, DOI 10.1112/jlms/s2-28.3.461. MR724715(85i:16036)

[4] Klaus Bongartz, A geometric version of the Morita equivalence, J. Algebra 139 (1991), no. 1, 159-171, DOI 10.1016/0021-8693(91)90288-J. MR.1106345 (92f:16008)

[5] Klaus Bongartz, Minimal singularities for representations of Dynkin quivers, Comment. Math. Helv. 69 (1994), no. 4, 575-611, DOI 10.1007/BF02564505. MR1303228(96f:16016)

[6] Klaus Bongartz, On degenerations and extensions of finite-dimensional modules, Adv. Math. 121 (1996), no. 2, 245-287, DOI 10.1006/aima.1996.0053. MR1402728(98e:16012)

[7] Giovanni Cerulli Irelli, Evgeny Feigin, and Markus Reineke, Quiver Grassmannians and degenerate flag varieties, Algebra Number Theory 6 (2012), no. 1, 165-194, DOI 10.2140/ant.2012.6.165. MR.2950163

[8] G. Cerulli Irelli, E. Feigin, and M. Reineke, Desingularization of quiver Grassmannians for Dynkin quivers, Adv. Math. 245 (2013), 182-207, DOI 10.1016/j.aim.2013.05.024. MR.3084427 
[9] William Crawley-Boevey, Normality of Marsden-Weinstein reductions for representations of quivers, Math. Ann. 325 (2003), no. 1, 55-79, DOI 10.1007/s00208-002-0367-8. MR1957264 (2004c:16017)

[10] Evgeny Feigin and Michael Finkelberg, Degenerate flag varieties of type A: Frobenius splitting and BW theorem, Math. Z. 275 (2013), no. 1-2, 55-77, DOI 10.1007/s00209-012-1122-9. MR.3101796

[11] D. Hernandez, B. Leclerc, Quantum Grothendieck rings and derived Hall algebras, Preprint 2011, arXiv:1109.0862

[12] A. Kirillov and J. Thind, Coxeter elements and periodic Auslander-Reiten quiver, J. Algebra 323 (2010), no. 5, 1241-1265, DOI 10.1016/j.jalgebra.2009.11.024. MR2584955(2011e:20060)

[13] G. Lusztig, On quiver varieties, Adv. Math. 136 (1998), no. 1, 141-182, DOI 10.1006/aima.1998.1729. MR.1623674 (2000c:16016)

[14] Lieven Le Bruyn and Claudio Procesi, Semisimple representations of quivers, Trans. Amer. Math. Soc. 317 (1990), no. 2, 585-598, DOI 10.2307/2001477. MR958897 (90e:16048)

[15] B. Leclerc, P. Plamondon, Nakajima varieties and repetitive algebras, Preprint 2012, arXiv:1208.3910

[16] Claus Michael Ringel, Tame algebras and integral quadratic forms, Lecture Notes in Mathematics, vol. 1099, Springer-Verlag, Berlin, 1984. MR774589 (87f:16027)

Mathematisches Institut, Universität Bonn, Bonn, Germany 53115

E-mail address: cerulli.math@googlemail.com

Department of Mathematics, National Research University Higher School of Economics, Russia, 117312, Moscow, Vavilova Str. 7 - and - Tamm Department of Theoretical Physics, Lebedev Physics Institute, Russia

E-mail address: evgfeig@gmail.com

Fachbereich C - Mathematik, Bergische Universität Wuppertal, D - 42097 Wuppertal, GERMANY

E-mail address: reineke@math.uni-wuppertal.de 